\title{
Design of a Quench Protection System for a Coated Conductor Insert Coil
}

\author{
D. Uglietti and C. Marinucci
}

\begin{abstract}
Coated conductors are promising material for the construction of high field magnets, but the slow propagation velocity of the normal zone makes the protection against quenches a critical issue. The quench protection system for a small insert coil under development at CRPP has been studied. The voltage drop and the hot spot temperature during the quench have been estimated using analytical expressions; the cross section of the copper stabilizer, the voltage threshold and the time constant were set as parameters. Detailed calculations have been carried out using the Cryosoft ${ }^{\mathrm{TM}}$ code THEA. The copper cross section available from the tape manufacturer is enough to ensure that the hot spot temperature remains below $200 \mathrm{~K}$, but only in conjunction with a sensitive quench detection system (voltage threshold lower than $100 \mathrm{mV}$ ) and a fast dumping time (time constant shorter than $100 \mathrm{~ms}$ ). These requirements can be fulfilled in the small insert coil under construction, because the inductance is about $50 \mathrm{mH}$ and because the coil can be quickly discharged independently from the main magnet. The passive protection circuit consists of dump resistor and diodes. In case of longer time constants (few seconds), a larger copper cross section (comparable to the one used in low temperature superconducting magnets) is required in order to maintain the temperature within safe values.
\end{abstract}

Index Terms-Superconducting coil, coated conductor, quench, stability.

\section{INTRODUCTION}

$\mathrm{H}$ IGH temperature superconducting (HTS) materials are considered for the construction of high field magnets in replacement of low temperature superconducting (LTS) materials. The first HTS insert coils were made with Bi based wires and tape [1-3], while the R\&D effort is now focused on Rare Earth based coated conductors. Coated conductors are promising material, because they provide large engineering critical current densities even at high field and high tensile stress. Progress in winding technology has been achieved in the last few years [4-5], but few works have been done on the quench detection and protection in coated conductor coils. The main problem concerning the protection of HTS coils is the slow normal zone propagation velocity (NZPV), which makes it extremely difficult to detect the quench in time. An

Manuscript received 12 September 2011. This work was partly financially supported by the Swiss National Science Foundation (SNF) under contract number 200021_126501.

D. Uglietti and C. Marinucci are with EPFL-CRPP, Fusion Technology, CH-5232 Villigen PSI (phone: +41 56310 5820; fax: +41 56 3103729; email: davide.uglietti@psi.ch). experimental study of the quench behavior of a Bi2212 coil has been presented in [6]: despite the slow NZPV of few $\mathrm{cm} / \mathrm{s}$, the coil could withstand several quenches without damage. Quench experiments have been carried out on various YBCO model coils [7-10], in order to study the NZPV, the minimum quench energy (MQE) and the general physics of the quench process. In summary, in a small insert coil (few Tesla) operated at $4.2 \mathrm{~K}$, the NZPV is expected to be between $0.1 \mathrm{~m} / \mathrm{s}$ and $1 \mathrm{~m} / \mathrm{s}$, while the temperature margin varies from $10 \mathrm{~K}$ (coil ends) to $30 \mathrm{~K}$ (coil mid-plane); the difference is due to the angular dependence of the critical current (anisotropy). For comparison, the NZPV in LTS coils is several meters per second, while the temperature margin is between 1 and $3 \mathrm{~K}$ in NbTi coils and from $2 \mathrm{~K}$ to $10 \mathrm{~K}$ in $\mathrm{Nb}_{3} \mathrm{Sn}$.

The NZPV is a material property for given field and temperature, and cannot be varied; the only free parameters that can be optimized are the voltage threshold at which the protection system is activated, the coil time constant and the cross section of the copper stabilizer. The influence of these three parameters on the hot spot temperature is studied for a small insert coil, which is currently under development at the Center for Research on Plasma Physics (CRPP). The goal is to evaluate the voltage threshold value, the time constant and the amount of copper required for a safe operation of the insert coil.

\section{QUENCH SIMULATIONS}

The insert coil under development at CRPP should provide $4 \mathrm{~T}$ in a background field of $12 \mathrm{~T}$ generated by a commercial LTS magnet. The coil height, inner and outer diameters are respectively $122 \mathrm{~mm}, 44 \mathrm{~mm}$, and $68 \mathrm{~mm}$. The inductance is about $50 \mathrm{mH}$ and the stored energy is about $1800 \mathrm{~J}$. The insert coil is located in the same LHe bath with the LTS magnet; the insert will not be connected in series with the existing LTS magnet, but it will be powered by an independent power supply. Details regarding design and construction are reported elsewhere [11]. It is planned to use a passive quench protection (resistor), with no heater, and a simple analog circuit to control the power supply.

Coated conductor from Superpower has been selected for the coil construction. The standard SCS4050 tape is stabilized with electroplated copper, whose cross section is about $0.16 \mathrm{~mm}^{2}$; as an option, a thicker stabilizer (up to $0.4 \mathrm{~mm}^{2}$ ) can also be obtained. The residual resistivity ratio (RRR) was measured on a short tape $(50 \mathrm{~mm})$. The tape was prepared by easily removing the electroplated copper on the YBCO side 
and the YBCO layer with a sharp scalpel; the resistance of the remaining Hastelloy substrate and copper stabilizer was measured with a four wires technique. The RRR from room temperature to $4.2 \mathrm{~K}$ was about 60 (resistivity of the copper at $4.2 \mathrm{~K}$ was estimated to be about $3 \cdot 10^{-10} \Omega \mathrm{m}$ ).

If it is assumed that the operating current is $260 \mathrm{~A}$, the current density in the copper stabilizer during the quench may reach about $1600 \mathrm{~A} / \mathrm{mm}^{2}$ for a standard YBCO tape (copper cross section $0.16 \mathrm{~mm}^{2}$ ) and $650 \mathrm{~A} / \mathrm{mm}^{2}$ for the tape stabilized with $0.4 \mathrm{~mm}^{2}$ copper. For comparison, the current density in a Bi2212 coil [6], whose NZPV is close to the one of coated conductor, was about $420 \mathrm{~A} / \mathrm{mm}^{2}$; the current density in the copper stabilizer of commercial $\mathrm{Nb}_{3} \mathrm{Sn}$ wires used for NMR or laboratory coil is less than $300 \mathrm{~A} / \mathrm{mm}^{2}$. These values clearly show that coated conductors have the highest current density in the copper stabilizer, thus the temperature may attain dangerous levels during the quench process.

\section{A. Analytical calculations}

Analytical calculations are very effective to study qualitatively the effect of parameters on temperature, while in the next section numerical calculation will be used to study in detail the temporal evolution of the system. The quench process can be subdivided in two steps: 1) quench initiation (at constant current), during which the voltage increases till reaching a threshold for the quench detection; 2) energy dump (when the voltage overcomes the detection value): the power supply is switched off and the energy stored in the coil is dumped; coil current decreases exponentially with a time constant $\tau=L / R$, where $R$ is the dump resistance and $L$ is the coil inductance.

In a simple model, it is assumed that the current flows entirely in the copper stabilizer; the process is adiabatic, and no heat conduction along or across the tape is considered. With these assumptions the temperature will be overestimated, thus allowing for a margin. The following equation is used to describe the temperature evolution

$$
C \frac{d T}{d t}=\rho(T) J^{2}
$$

where $C$ is the thermal capacity, $T$ the hotspot temperature, $\rho$ the resistivity and $J$ the Joule heating. For a coated conductor, (1) can be written as:

$$
A_{H} C_{H} \frac{d T}{d t}+A_{C u} C_{C u} \frac{d T}{d t}=\frac{1}{A_{C u}} \rho(T) I_{o p}^{2},
$$

where $A_{H}$ and $A_{C u}$ are the Hastelloy and copper cross-section, $\rho$ is the copper resistivity, $C_{H}$ and $C_{C u}$ are the Hastelloy and copper temperature dependent thermal capacity and $I_{o p}$ is the operating current of the coil. By integration of (2) an implicit expression for the hotspot temperature, $T$, is obtained:

$$
\left\{A_{C u} A_{H} \int_{T_{i}}^{T} \frac{C_{H}(y)}{\rho(y)} d y+A_{C u}^{2} \int_{T_{i}}^{T} \frac{C_{C u}(y)}{\rho(y)} d y\right\}=\int_{0}^{t} I_{o p}^{2}(t) d t .
$$

The voltage drop across the normal section is

$$
V=I_{o p} \frac{v_{N Z} t}{A_{C u}} \rho(T)
$$

where $v_{N Z}$ is the NZPV, which is supposed to be $1 \mathrm{~m} / \mathrm{s}$; in the next section this value will be estimated.

First, the hotspot temperature increment before the quench detection (in (3) the current is constant) is studied. The hotspot temperature as a function of the copper cross section is plotted in Fig. 1, the voltage being a parameter; the curves have been calculated using (3) and (4). If the threshold level is low (for example less than $200 \mathrm{mV}$ ) then the hotspot temperature is almost constant irrespective of the copper cross section. Therefore, large copper cross sections do not bring any advantage. It should be pointed out that for small copper cross section the voltage rises very quickly: for example, the $200 \mathrm{mV}$ value is reached in less than $10 \mathrm{~ms}$. At large voltage threshold (for example $>0.5 \mathrm{~V}$ ) the hotspot temperature is very high if the copper cross section is small, thus large cross sections are required for a safe operation.

After quench detection, the current decreases exponentially, but the hotspot temperature continues to increase. Starting from an initial value of $60 \mathrm{~K}$, which is assumed to be the temperature before dumping, the hotspot temperature increment during the current exponential decay is plotted as a function of $\tau=L / R$ in Fig. 2. Even for very short time constant (less than $100 \mathrm{~ms}$ ) the $0.16 \mathrm{~mm}^{2}$ copper cross section may be too small to ensure that the temperature stays below $200 \mathrm{~K}$; lower hotspot temperatures are obtained with a modest increase of the copper cross section. If the time constant is longer than one second, very large copper cross section $\left(>1 \mathrm{~mm}^{2}\right)$ are needed.

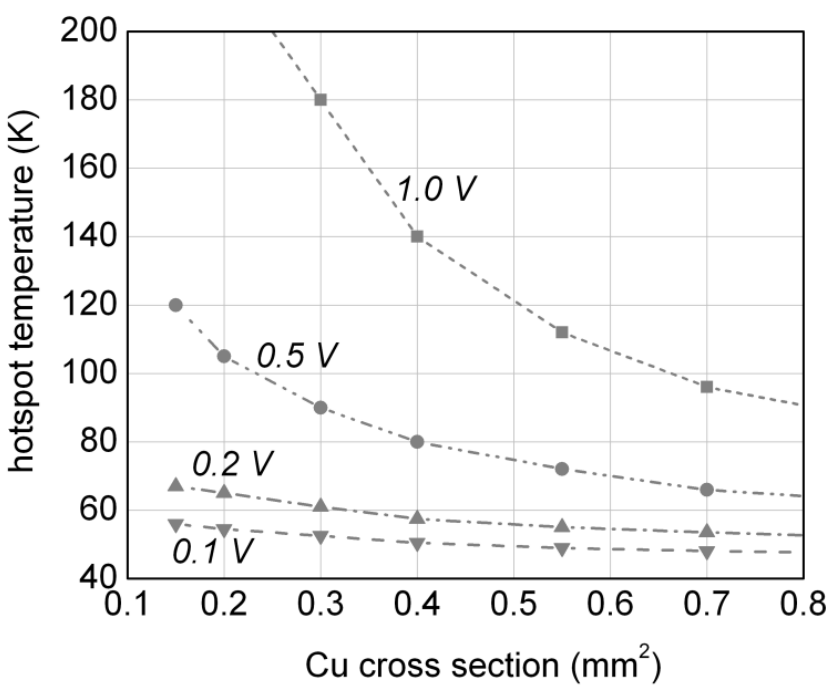

Fig. 1. Hotspot temperature versus copper cross section before quench detection. The voltage drop across the normal section is also indicated. 


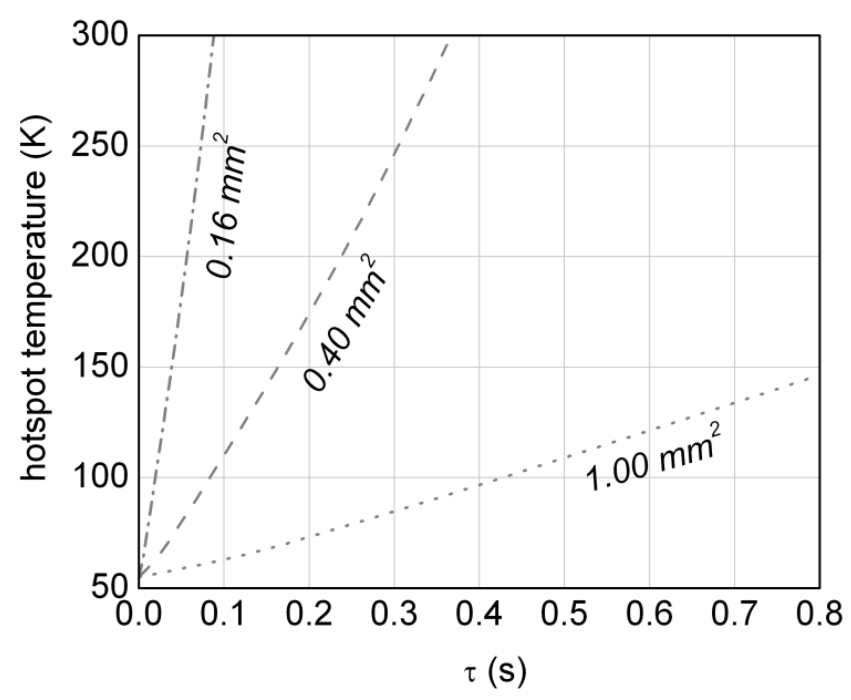

Fig. 2. Hotspot temperature versus time constant during discharging, for various copper cross sections.

In the insert coil considered in this work, both $100 \mathrm{mV}$ threshold and $100 \mathrm{~ms}$ of time constant could be reasonably obtained, thus it may be expected that the $0.4 \mathrm{~mm}^{2}$ copper cross section is large enough to maintain the hotspot temperature below $200 \mathrm{~K}$. Instead, the standard $0.16 \mathrm{~mm}^{2}$ cross section may be too small to protect the coil: the choice of $0.4 \mathrm{~mm}^{2}$ allows a margin both on the detection voltage and on the time constant.

In large coils, because of the larger inductance, the time constant may be longer than one second, thus copper section of $1 \mathrm{~mm}^{2}$ or larger are needed, as it was found in [12], i.e. a value similar to the one used in commercial $\mathrm{Nb}_{3} \mathrm{Sn}$ wires. In summary, the main effect of large copper sections is to slow down the time scale of the quench process.

\section{B. Numerical calculations}

The objective is to obtain an accurate simulation of the tape temperature and voltage evolution during the quench, considering copper cross section of 0.16 and $0.40 \mathrm{~mm}^{2}$, i.e. the standard and the maximum cross sections that are available from tape manufacturer. Voltage detection level and dumping time $(\tau=L / R)$ are chosen to be consistent with the design of the YBCO insert coil. The simulation is carried out using the CryoSoft $^{\mathrm{TM}}$ code THEA [13]. The scaling law for coated conductors can be found in [14]. The process is adiabatic and thermal conduction take place only along the longitudinal direction of the tape. The quench is supposed to occur at the coil mid-plane, because it is more dangerous and difficult to protect, the temperature margin being the largest (over $30 \mathrm{~K}$ ) and the propagation velocity being the slowest. The tape critical current at coil mid-plane is about $650 \mathrm{~A}$ ( $16 \mathrm{~T}$ parallel to the wide face of the tape), while the operating current is $260 \mathrm{~A}$.

The quench is induced by a heat pulse along $20 \mathrm{~mm}$ of tape, lasting $100 \mathrm{~ms}$ from $\mathrm{t}=0 \mathrm{~s}$. For the tape with $0.16 \mathrm{~mm}^{2}$ copper, the heat pulse was $35 \mathrm{~W} / \mathrm{m}$, while for the tape with $0.40 \mathrm{~mm}^{2}$ it was $70 \mathrm{~W} / \mathrm{m}$. At lower heating powers the tape recover to the superconducting state, as it was found experimentally in [10]. The quench is driven by the exponential $n$ factor, which is assumed to be 20 . In the simulation, when the voltage reaches the quench detection limit $(100 \mathrm{mV})$, the current is decreased exponentially with a time constant of $100 \mathrm{~ms}$; the dumping resistance is supposed to be about $0.5 \Omega$, which ensures that the voltage is below $200 \mathrm{~V}$ during the discharge.

In Fig. 3 the tape temperature and the current are plotted as a function of time for the tape with $0.16 \mathrm{~mm}^{2}$ copper. The voltage reached $100 \mathrm{mV}$ at $\mathrm{t}=230 \mathrm{~ms}$, i.e. less than $50 \mathrm{~ms}$ after the temperature took off and $130 \mathrm{~ms}$ after the heat injection. The maximum temperature was about $230 \mathrm{~K}$.

In Fig. 4 the tape temperature and the current are plotted as a function of time for the tape with $0.40 \mathrm{~mm}^{2}$ copper. The voltage reached $100 \mathrm{mV}$ at $t=460 \mathrm{~ms}$, i.e. about $150 \mathrm{~ms}$ after the temperature take off and $350 \mathrm{~ms}$ after the heat injection. Therefore, in the tape with $0.40 \mathrm{~mm}^{2}$ copper, the quench process is slower than in the tape with $0.16 \mathrm{~mm}^{2}$, as it was expected from analytical calculations. The maximum temperature is also lower than in the $0.16 \mathrm{~mm}^{2}$ case, being about $130 \mathrm{~K}$. The NZPV is about $0.3 \mathrm{~m} / \mathrm{s}$, and the normal zone length is about $15 \mathrm{~cm}$ at the end of the current dump (the initial length was about $2 \mathrm{~cm}$ ).

The only disadvantage associated with the tape with $0.40 \mathrm{~mm}^{2}$ copper could be the lower engineering current density; nevertheless, the value will be $300 \mathrm{~A} / \mathrm{mm}^{2}$ (including the insulation), which is about two times larger than the one obtained in Bi-based insert coils. In the choice of the copper cross section, we fully profit of the possibility to power the insert coil with a separate power supply. When the simulation in Fig. 4 is repeated assuming a time constant $\tau=1 \mathrm{~s}$ instead of $100 \mathrm{~ms}$, the temperature will peak at over $800 \mathrm{~K}$. Therefore a much larger copper cross section would be needed to protect the insert coil if it had been operated in series with the LTS magnet.

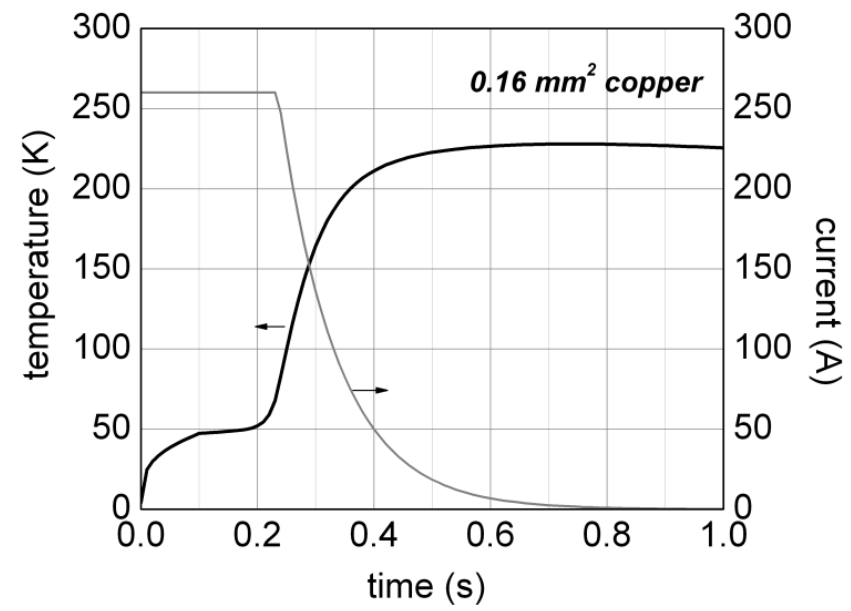

Fig. 3. Evolution of tape temperature and current during the entire quench process for the tape with $0.16 \mathrm{~mm}^{2}$ copper stabilizer. Current dump started when the voltage drop reached $100 \mathrm{mV}$. 


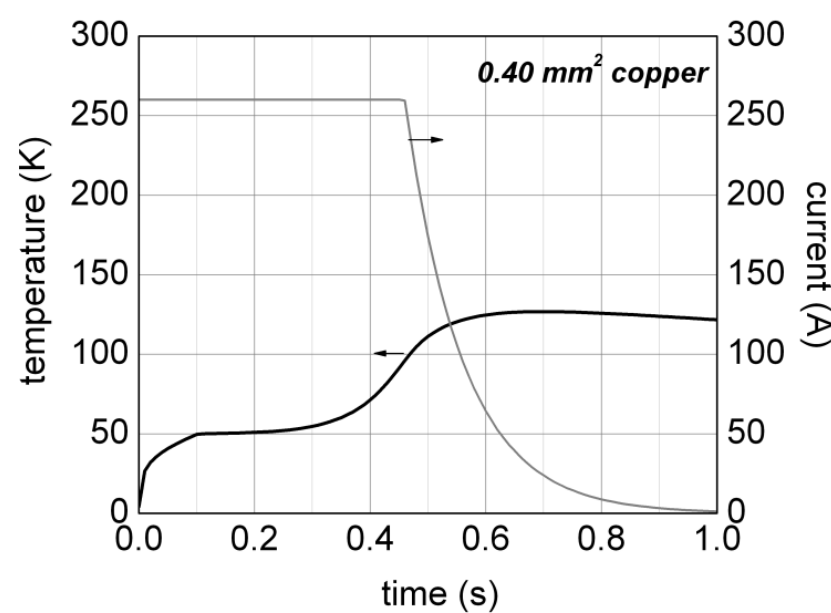

Fig. 4. Evolution of tape temperature and current during the entire quench process for the tape with $0.40 \mathrm{~mm}^{2}$ copper stabilizer. Current dump started when the voltage drop reached $100 \mathrm{mV}$.

\section{PROTECTION SCHEME OF THE YBCO INSERT COIL}

The YBCO insert coil will be sub-divided in two concentric coils, voltage taps will be attached to each sub-coils and connected so that the inductive contribution is canceled. It may also be possible to use a small copper coils on one current lead in order to cancel the inductive contribution. The voltage drop across the coil will control a digital input on the power supply: the power supply will be switched-off (time response is few $\mathrm{ms}$ ) when the voltage exceeds the voltage threshold value $(<100 \mathrm{mV})$ and the energy is dumped into a resistor in parallel with the insert coil.

In case the LTS magnet is quenched, the detection system of the insert coil should also be activated, and the insert coil will be fully discharged much faster than the LTS magnet. Therefore, during the discharge of the LTS magnet, the insert coil is connected in series with the protection resistor, thus the voltage induced by the LTS coil in the insert coil will produce only a modest current.

\section{CONCLUSION}

The quench behavior of a small insert coil built with coated conductor has been studied by analytical and numerical methods. It was found that it is possible to use the standard tape from Superpower (with optional $\mathrm{Cu}$ cross section of $0.4 \mathrm{~mm}^{2}$ ) only in conjunction with a sensitive quench detection system (less than $100 \mathrm{mV}$ ) and fast dumping circuit ( $\tau$ shorter than $100 \mathrm{~ms}$ ). More in details, it takes less than $150 \mathrm{~ms}$ before the voltage reaches the threshold value $(100 \mathrm{mV})$; during the exponential decay, which will last few hundreds of ms (time constant is about $100 \mathrm{~ms}$ ), the tape temperature will not exceed $130 \mathrm{~K}$. The NZPV has been estimated to be less than $0.3 \mathrm{~m} / \mathrm{s}$ and the length of normal zone region at the end of the current dump is less than $15 \mathrm{~cm}$ (initial length of the normal zone was $2 \mathrm{~cm})$. In case the copper cross section is $0.16 \mathrm{~mm}^{2}$, the maximum tape temperature will be about $230 \mathrm{~K}$ and the whole quench process will be much faster. The tape with $0.4 \mathrm{~mm}^{2}$ copper cross section has been chosen because it ensures a larger margin and is readily available from the manufacturer.

The choice of $0.4 \mathrm{~mm}^{2} \mathrm{Cu}$ cross section relies on the fact that the insert coil is not connected in series with the LTS magnet, thus it can be quickly discharged (time constant shorter than $100 \mathrm{~ms}$ ), independently from the time constant of the LTS magnet (longer than one second). If the insert coil was connected in series with the LTS magnet, or in case of much larger coated conductor coils, the time constant would be longer than one second, thus a much larger copper crosssection would be required to maintain the temperature below $200 \mathrm{~K}$.

The passive quench protection envisaged for the insert coil includes a dumping resistor and a simple analog circuit to control the power off of the coil.

\section{ACKNOWLEDGMENT}

The authors thank the Paul Scherrer Institute (PSI) for the technical support.

\section{REFERENCES}

[1] T. Kiyoshi et al. "Generation of $23.4 \mathrm{~T}$ Using Two Bi-2212 Insert Coils," IEEE Trans. Appl. Supercond., vol. 10, no. 2, pp. 472-477, Mar. 2000.

[2] H. W. Weijers et al., "The generation of $25.05 \mathrm{~T}$ using a $5.11 \mathrm{~T}$ $\mathrm{Bi}_{2} \mathrm{Sr}_{2} \mathrm{CaCu}_{2} \mathrm{O}_{\mathrm{x}}$ superconducting insert magnet," Supercond. Sci. Technol., vol. 17, pp. 636-644, 2004.

[3] M. Beckenbach et al., "Manufacture and test of a 5 T Bi-2223 Insert Coil," IEEE Trans. Appl. Supercond., vol. 15, no. 2, pp. 1484-1487, Jun. 2005.

[4] W. D. Markiewicz et al., "33.8 Tesla with a YBa2Cu3O7-x superconducting test coil”, AIP Conference Proceedings, vol. 1218, pp.225-230, 2010.

[5] D. Uglietti, S. Choi and T. Kiyoshi, "Design and fabrication of layerwound YBCO solenoids", Physica C, vol. 470, pp. 1749-1751, 2010

[6] U. P. Trociewitz et al. "Quench studies on a layer-wound $\mathrm{Bi}_{2} \mathrm{Sr}_{2} \mathrm{CaCu}_{2} \mathrm{O}_{\mathrm{x}} / \mathrm{AgX}$ coil at $4.2 \mathrm{~K}$ ”, Supercond. Sci. Technol., vol. 21, pp. 025015, 2008.

[7] H. Song, K. Gagnon and J. Schwartz, "Quench behavior of conductioncooled $\mathrm{YBa}_{2} \mathrm{Cu}_{3} \mathrm{O}_{7-\delta}$ coated conductor pancake coils stabilized with brass or copper", Supercond. Sci. and Tech., vol. 23, pp. 065021, 2010.

[8] F. Trillaud et al., "Quench Behaviour, Quench Protection of a YBCO Test Coil Assembly”, IEEE Trans. Appl. Supercond., vol. 18, no. 2, pp. 1329-1332, June 2008.

[9] W. Wang, U. P. Trociewitz and J. Schwartz, "Critical current degradation of a short $\mathrm{YBa}_{2} \mathrm{Cu}_{3} \mathrm{O}_{7-\delta}$ coated conductor due to an unprotected quench", Supercond. Sci. Technol., vol. 24, pp. 035006, 2011.

[10] H. Song and J. Schwartz, "Stability and Quench Behavior of $\mathrm{YBa}_{2} \mathrm{Cu}_{3} \mathrm{O}_{7-\mathrm{x}}$ Coated Conductor at $4.2 \mathrm{~K}$, Self-Field", IEEE Trans. Appl. Supercond., vol. 19, no. 5, pp. 3735-3743, October 2009.

[11] R. Wesche, et al. "Development of Magnet Technologies for HTS Insert Coils", to be published in Physics Procedia, 2011.

[12] S. Hahn, J. Bascunan, W. Yao and Y. Iwasa, "Two HTS options for a $600 \mathrm{MHz}$ insert of a $1.3 \mathrm{GHz}$ LTS/HTS NMR magnet: YBCO and BSCCO”, Physica C, vol. 470, pp. 1721-1726, 2010.

[13] L. Bottura, C: Rosso and M. Breschi, "A General Model for Thermal, Hydraulic and Electric Analysis of Superconducting Cables", Cryogenics, vol. 48, pp. 617-626, 2000.

[14] C. Marinucci, L. Bottura, M. Calvi and R. Wesche, "Quench analysis of a high-current forced-flow HTS conductor model for fusion magnets", IEEE Trans. Appl. Supercond., vol. 21, no. 3, pp. 2445-2448, Jun. 2011. 